Theology and Science, Vol. 9, No. 3, 2011

\title{
Celestial Aesthetics: Over Our Heads and/or in Our Heads
}

\section{HOLMES ROLSTON, III}

I am the daughter of earth and water, And the nursling of the sky.

Percy Bysshe Shelley, "The Cloud" 1820

Abstract Looking at the night sky, we may seem cosmic dwarfs, overwhelmed with a sense of otherness, abyss, lostness. But humans alone enjoy such celestial awe. We can move to a sense of the beholder's celestial ancestry and ongoing relatedness in "our cosmic habitat." That account joins aesthetics with mathematics, finds dramatic interrelationships gathered under "the anthropic principle," and considers meteorological aesthetics. The wonder is as much this Homo sapiens with mind enough to search the universe. What is out there is inseparably linked with what is down here. We are at home in the universe. The glory is both over our heads and in our heads.

Key words: Celestial aesthetics; Awe; Lostness; Mathematics; Cosmic habitat; Meteorological aesthetics; Clouds; Atmosphere; Mind and cosmos

\section{Humans lost in the stars}

Once up a time, my ancestors gazed at the stars, earth below, heavens above, the firmament on high, and wondered before an awesome and celestial vista, the abode of angels, gods, God. In more recent centuries, astrophysics discovered that humans, on a small planet, inhabited a fantastically huge clockwork, rockwork universe, a mechanism of matter in energetic motion. The heavens were fabulously big but no longer seemed heavenly. There was celestial decay. In the sky was more dirt, mountains on the moon, asteroids in deep space. Humans were cosmic dwarfs, lost out there in the stars. E.A. Burtt lamented:

The world that people had thought themselves living in — $a$ world rich with colour and sound, redolent with fragrance, filled with gladness, love and beauty, speaking everywhere of purposive harmony and creative ideals-was now crowded into minute corners in the brains of scattered organic beings. The really important world outside was a world hard, cold, colourless, silent, and dead; a world of quantity, a world of mathematically computable motions in mechanical regularity. ${ }^{1}$

ISSN 1474-6700 print/ISSN 1474-6719 online/11/030273-13

(C) 2011 Center for Theology and the Natural Sciences

DOI: $10.1080 / 14746700.2011 .587663$ 


\section{Theology and Science}

Tennyson contemplated human life placed in such vastness: "What is it all but a trouble of ants in the gleam of a million million of suns?"2 Karl Jaspers found Earth "a minute grain of dust in the universe... in an out-of-the-way corner. ... The fundamental fact of our existence is that we appear to be isolated in the cosmos. We are the only articulate rational beings in the silence of the universe .... This is the place, a mote in the immensity of the cosmos, at which being has awakened with man." ${ }^{3}$ When cosmologist Steven Weinberg got back to the first three minutes, he concluded famously: "The more the universe seems comprehensible, the more it also seems pointless."

Is such a vast, silent, pointless universe today the settled framework of celestial aesthetics? Looking up at the stars, are we overwhelmed with our insignificance? Still reeling from that, we puzzle that, cosmic dwarfs though we are, ants in vastness, humans alone enjoy such celestial wonder.

Celestial aesthetics does start with an overwhelming sense of otherness, distance, abyss, disinterest, lostness. But that is not where I end up. I want to move to a sense of participation, of the beholder's celestial ancestry and ongoing relatedness. I wish to place celestial aesthetics in a science-based framework, a cosmological aesthetics, but the astrophysics has been changing. I intend to soften the hard-edged lines between the objective and the subjective, between the objects (such as stars in the sky) and the subject (experiencing beholder). When I look up at the sky, I need to tell a story that moves from there to here.

That requires both the immediate experience of looking upward and mediated experiences looking through telescopes, astronomical theories, and worldviews. I see what Martin Rees calls "our cosmic habitat." 1 am rejecting the limited scope of aesthetics as beauty perceptible to the senses, expanding aesthetics to include beauty sensed by the reasoning mind, that is, to concepts as well as to percepts, to concepts synthesized with percepts.

\section{Mathematics and aesthetics}

The twinkling stars are out there, sprinkled through the reaches of space. But they cannot have beauty without us - so we have been taught to think-because beauty is in the eye of beholder. The universe without such beholders is beautyless; celestial aesthetics is a state of mind. The stars sparkle, only apparently; yet the capacity for aesthetic experience appears and sparkles in humans. Like rainbows, is celestial aesthetics all an aesthetically pleasing illusion? No, looking at those stars, I find some complementarity in aesthetics, some blending of what is up there over my head and what is down here in my head.

I argue with an analogy to mathematics. The mathematical properties are there in nature (conservation of energy or charge, radial symmetry), but the discipline of mathematics (equations balancing energy, charge, tracking a symmetry) is a human creation in interaction with what there is in nature. Similarly, the aesthetic properties (form, symmetry) are out there; the aesthetic experience arises relationally. The mathematical dimensions of nature, found when mathematics is applied to natural phenomena, are often also aesthetic, as evidenced in physics, 
astronomy, chemistry and crystallography. Indeed, even in pure mathematics, often celebrated as supremely rational, there is already an aesthetic dimension. The equations are logically rigorous, calculating; they are simultaneously formal, symmetrical, balanced, cohesive, integrating. A proof of a theorem is not only right; it is pleasing.

Looking at the sparkling stars, we might conclude that beauty is not objectively present in nature; it is in the eye of the beholder. However, at this point, we have to distinguish two sorts of aesthetic qualities: aesthetic capacities, capacities for felt experience that are only in beholders; and aesthetic properties, which lie objectively in natural things. The experience of beauty does arise in the beholder, but what is this experience of? It is of form, structure, integrity, order and process. These events are there before humans arrive, indeed often long before, since we look back in time as we look outward. When we value them aesthetically, our experience is being superimposed on natural properties.

In a sense, systemic nature even has an aesthetic power, since it is able to produce aesthetic properties, even though nature does not have-until it produces this in humans! - the capacity for felt aesthetic experience. When humans arrive and are stimulated by astronomical nature, we are valuing a constructive nature that we are discovering, sometimes more than we are projecting our values onto nature. Nature carries aesthetic properties objectively, and these are ignited in the subjective experience of the arriving beholder. There is aesthetic stimulation, for instance, in the sense of abyss overlooking a canyon, or staring into space. Similarly, with the fury of a hurricane at sea. That experience is in the beholder, but the abyss and fury (the aesthetic properties) are not in the mind, they are in nature. Human emotions track the motions of nature.

The world is beautiful in something like the way it is mathematical. Neither aesthetic experience nor mathematical experience exist prior to the coming of humans (setting aside for the present the possibility of animal aesthetic experience). Mathematics and aesthetics are human constructs; they come out of the human head and are used to map the world. This is also true with theories in the natural sciences, with lines of latitude and longitude, with contours on maps. Regression lines (averaging out trends in data and correlating variables) and centers of gravity, as figured into equations, do not exist in nature. But these inventions succeed in helping humans to find their way around in the world because they map form, symmetry, harmony, distribution patterns, causal interrelationships, order, unity, diversity, and so on, discovered to be actually there. Since time immemorial, the regularities of the heavens, sun, moon and stars have given us our primary senses of measuring time-days; months, equinox, solstice, years. The vault above us has been important in developing our capacities for time, number, measurement, although there is no sense of passing time, no calculating with numbers, nothing taking measurements on the moon.

It is true to say that the world is objectively mathematical and, at the same time, to say that mathematics is a subjective creation of the human mind. John A. Wheeler, a physicist and cosmologist, exclaims, "This is a world of pure 


\section{Theology and Science}

mathematics and when we penetrate to the bottom of it, that's all it will be.' ${ }^{6}$ Eugene P. Wigner, a physicist and mathematician, contends "that the enormous usefulness of mathematics in the natural sciences is something bordering on the mysterious and that there is no rational explanation for it... . The miracle of the appropriateness of the language of mathematics for the formulation of the laws of physics is a wonderful gift which we neither understand nor deserve. ${ }^{7}$ For these cosmologists, this is a "matheomorphic" universe.

Mathematical properties are really there, though mathematical experience awaits the human coming - and analogously with aesthetic properties and capacities. It is thus no accident that mathematicians are invariably among those who find the world aesthetically delightful in its symmetries, curves, patterns. Think, for example, of the majestic spiral Whirlpool Galaxy, M51.

Nor is it simply that the mathematics is analogically parallel to the aesthetics; rather the mathematics itself has an aesthetic dimension. Paul Dirac wrote:

It seems then that if one is working from the point of view of getting beauty in one's equations, and if one has a really sound insight, one is on a sure line of progress. If there is not complete agreement between the results of one's work and experiment, one should not allow oneself to be too discouraged, because the discrepancy may well be due to minor features that are not properly taken into account and that will get cleared up with further development of the theory. ${ }^{8}$

John Polkinghorne, a student of Dirac's, recalls that once in class Dirac "was asked what was his fundamental belief. He strode to a blackboard and wrote that the laws of nature should be expressed in beautiful equations." ${ }^{9}$ Commenting on the relationship between mathematics and physics, Dirac said: "What makes the theory of relativity so acceptable to physicists in spite of its going against the principle of simplicity is its great mathematical beauty. ${ }^{10}$

Judith Wechsler concluded: "The search in science for models that illuminate nature seems to parallel certain crucial processes in art." "When scientists ... reflect on their work, the development of concepts, and the theories that expound them, it is evident that intuition and aesthetics guide their sense of 'this is how it has to be,' their sense of rightness." 11

Here a critic may object that this expanding of aesthetics to include beauty sensed by the reasoning mind, synthesizing percepts with concepts, finding complementarity between mathematics and aesthetics, has forgotten that aesthetics is perceptual. Indeed, celestial aesthetics is visual. There is nothing to hear, taste, touch, smell. And the visual is monochrome, all white, with at most a tinge of red or blue here and there.

What then should we make of multi-wavelength astronomy? If we add colorenhanced images, is not the color reflecting symmetries out of the visual color range, but still objectively there? Color-enhanced images do pick up form and convert it to color, and when we enjoy that color, we are tracking patterns in the heavens. Think again of the Whirlpool Galaxy, perhaps you have seen images of it in enhanced color, white, blue, and red, where further dimensions of its form and symmetry are revealed. 


\section{Wild sky: Order and chaos}

Yet such celestial aesthetics gets challenged. When we make it complementary to mathematics, there is formal beauty, symmetry, elegance. Here the critic replies: Such mathematics is abstracted from nature; it does not catch the real, more broken world. When I look up at the stars, the heavens are ordered, yes. But the heavens too, are wild. There is order. Equally, there is disorder, chaos. Looking up at the starry night, there is no evident structure; there is only a jumble of stars. There is no order, just stars thrown about. The Double Cluster in Perseus does show two galactic clusters, but they are just that: clusters with nothing aesthetic about their scattering. If I turn to the moon, there is nothing mathematical about the way the craters on the moon are distributed. The meteors and asteroids that caused them perhaps have orbits that can be calculated with celestial mechanics, but the distribution of such bodies in space is in helter-skelter disarray. The ancient astronomers were too much inclined to see circles and epicycles superimposed on these circles; they were led astray by their desires to find order.

When we look up at the stars there is no pattern. Today, we are almost amused at the way the ancients fancied various constellations there. At night, we no longer admire the Orion as a hunter, any more than by day do we admire a cumulus cloud as a basket of washing. Nor do we-the educated among us in the West at least - consult astrological charts as we look above. At Stonehenge we sense some of those primitive emotions that moved the ancients to watch the stars, but nobody any longer tries to "hitch their wagon to a star." We do not aspire to be in the "seventh heaven." The stars have no orienting directions, even if each one follows lawlike paths.

Perhaps we have not so much given up star-gazing, as transformed it. Instead, we must have more accurate information in our minds, essentially astronomical information, to see what is there and respond to it aesthetically. If so, then when we abstract out the celestial mechanics and admire the equations, is even that beauty nothing but a selective reflection of our mind? The skies do in fact mirror these equations in some of their processes, but celestial events are otherwise mostly erratic, pointless, random. There is no significance there astrologically, nor-we may next worry - even mathematically. In applying mathematics to the world, whether in astrophysics or microphysics, much is left out, and mathematics is to that extent stylized and crude as a description of more complex and idiosyncratic natural processes.

Albert Einstein famously disliked disorder in his universe: "I ... am convinced that He (God) is not playing at dice."12 Einstein concluded that "the eternal mystery of the world is its comprehensibility."13 Disorder left his universe too unsimple, too irrational. Physicists cannot completely metricize the individual quantum event; it defies mathematical specification in its concreteness. That is why Einstein disliked the indeterminacy in quantum theory. Here is Werner Heisenberg in conversation with Einstein:

You may object that by speaking of simplicity and beauty I am introducing aesthetic criteria of truth, and I frankly admit that I am strongly attracted by the simplicity 


\section{Theology and Science}

and beauty of the mathematical schemes which nature presents us. You must have felt this too: the almost frightening simplicity and wholeness of the relationship, which nature suddenly spreads out before us. ${ }^{14}$

Yes, there are the simple mathematical formulations that Heisenberg admired; but the astronomical realms are also "wild." With this significant modifier, some perspectives shift. "Wild" gets at those levels in nature where there is mixed stability and spontaneity. There is a mixture of order and chaos. The reference to "wild" is not ordinarily to molecular or atomic scales. Scientists do not usually think of a single carbon atom as being wild, nor do they describe crystal structures as being wild. Crystal structures are too orderly. "Wild" retains some of the "uncontrolled" or "unlawful" or "erratic" or "spontaneously autonomous" elements.

Originally, the reference "wild" is to nature that is outside human plan and control. But within that domain, the reference continues to nature outside simple lawlike patterns. Humans do not control these events; neither are they completely specified naturally. Geomorphological and climatological processes qualify as "wild" better than simple physical and chemical ones. Antarctica is wild. We think of a moonscape as being wild; rocks and debris are scattered there; meteors have left their impact. But eclipses of the moon can be predicted to within microseconds for centuries ahead; the clockwork regularity overwhelms the spontaneity. The process is too automatic to catch what we mean by "wild." "Mechanical" is not a synonym for "wild." "Wild" needs more evident turbulence and ferment, more autopoiesis.

Many processes in these wild domains may be determinate, but there will be the intersection of causally unrelated lines, producing novelty and unpredicted events. Individual events rattle around in the statistics. Recent science accentuates genuine contingency, openness mixed with determinate laws. There is creative mixing of the ordered and the spontaneous. Galaxies are always the same and never the same, the same laws, similar patterns, and yet each different, because the galaxy processes are not simply determinate; there are chaotic elements.

Gravity is obeyed without exception throughout the universe. But Europa, Jupiter's largest moon, is in a gravitational tug of war of incredible dimensions, pulled in different directions by Jupiter and by the planet's other moons. This flexes the brittle outer surface into a criss-cross of dynamically changing straight and curved ridges, valleys, cracks, with bands of ice, generating heat that may melt lakes or oceans below. Saturn's little satellite, Enceladus, has so little gravity and orbits so deeply in Saturn's powerful gravity that there results a huge plume of ice-laden water that spouts from an overheated South Pole region. Both these moons are like no place else in our solar system, perhaps like no place else in the universe. ${ }^{15}$

The simply beautiful, the beautiful simple is too simple to produce interesting results. There needs to be more complexity; the complexity needs to have broken symmetries, without which there would be no differences, no complex processes, indeed none of the heavier elements, no planets, no evolution of life on Earth. In this less simple order, there is a mixture of order and disorder, of openness that introduces new possibility space. 
Leaving the well-ordered physics and mathematics, Frances Crick, discoverer of DNA, complains that biology has no "elegance." Organisms evolve happenstance structures and wayward functions that have no more overarching logic than the layout of the Manhattan subway system. ${ }^{16}$ We are inclined to think genetics more wild than crystallography, although they are equally processes in spontaneous nature. These genetic events are correlated with the contingencies of natural selection. Natural history wanders around; there is no similarity between the tracks of an evolving species on Earth and the tracks of an asteroid in the astronomical heavens. John Herschel (1792-1871), English mathematician, astronomer, and contemporary of Darwin, disliked natural selection as too messy, and called it Darwin's "law of higgledy-piggledy."17

Some think that, while the big-scale world of Darwin is happenstance and wayward, the biomolecular structures do have a certain elegance. James Watson, Crick's co-discoverer, thought just the opposite from Crick: he describes their discovery as "the spirit of an adventure characterized both by youthful arrogance and by the belief that the truth, once found, would be simple as well as pretty." ${ }^{18}$ A spiral is, after all, rather symmetrical and elegant, whether at molecular levels in DNA or galactic levels in the Whirlpool Galaxy. There is a certain simplicity to the spiral helix coding, only four cross links, triplet codons specifying some 20 amino acids. The genetic information coded on such a helix is, however, quite complex, enormously more so than anything in the heavens.

\section{Our celestial ancestry}

There is order and disorder in the heavens, and the outcome of this interplay brings us to think more deeply of relating what is out there to what is down here. I, the beholder, have a celestial ancestry and ongoing relatedness. If there were nothing out there, there would be nothing down here. First we may seem lost in the stars, but then again there are connections. John Barrow, mathematical cosmologist, surveys the universe: "Many of its most striking features its vast size and huge age, the loneliness and darkness of space-are all necessary conditions for there to be intelligent observers like ourselves."19

In the last half-century, cosmologists have found dramatic interrelationships between astronomical and atomic scales that connect to make the universe "userfriendly." These discoveries are commonly gathered under the name "the anthropic principle," a term introduced by Brandon Carter in 1974. The reference is to a series of observations about the values of the fundamental constants, the fundamental forces, the properties of particles, such as charge and mass, the nature of dynamic processes, the initial conditions. When these are figured into the theories of physics, the result in our universe appears to be

"fine-tuned" so as to enable the development of complex chemistries, which are requisite for life and self-conscious mind. What to make of this anthropic principle has since generated much debate, too complex to review as one contemplates the night sky. But those alerted to this debate no longer look at the 
heavens without wondering both about the sky and about what these anthropic relations might be.

I am looking out and looking back. If I could look all the way back, I would see the primordial big bang, which launched these heavens ongoing before my eyes in continuing expansion, at a critical rate. If the expansion rate of the universe had been a little faster or slower, then the universe would already have recollapsed or the galaxies and stars would not have formed. The various heavier elements (carbon, oxygen, sulphur, nitrogen, silicon, all of the elements heavier than hydrogen and helium) are forged in stellar furnaces in proportions that make later planets and life possible. The atoms in my body, in the ground beneath my feet, are fossil stardust. Above, we see twinkle, twinkle and realize it is from the cooking pots that make Earth and life on Earth possible.

On a clear night, I can see a few fuzzy patches that I know to be galaxies, and I think again of the vastness. Then I think: if the scale of the universe were much reduced (to galaxy size for instance), there would not have been enough time for stars to form and generate these elements. Paul Davies, a cosmologist, claims that we hit "the cosmic jackpot," a universe "just right for life." ${ }^{20}$ Roger Penrose, a mathematical physicist, concludes that ours is "an extraordinarily special Big Bang." ${ }^{21}$ Martin Rees concludes: "We should surely probe deeper, and ask why a unique recipe for the physical world should permit consequences as interesting as those we see around us." ${ }^{22}$

Thinking backward in time, still looking up at the night sky, my attention shifts to trees on the skyline of the mountain to the west. I hear a coyote howl. First I reel at being lost out there in the stars. But then I am brought back down to Earth. I begin to wonder if the astronomical start up is some kind of a set up for me and my world.

\section{Meteorological aesthetics}

Perhaps I have been too astronomical. When I look up at the heavens most of the time, I see no stars at all. Daytime, I see blue sky, and only one star, the sun, too brilliant to look at. The sky is the dominant source of light in nature. Shakespeare disliked clouds: "The more fair and crystal is the sky, the uglier seem the clouds that in it fly."23 But I really prefer a sky with some clouds, the most evident visual manifestation above us. Meteors are in the night sky, but meteorology is the weather, day and night (Gk., meteoros: high in the heavens). Celestial aesthetics includes meteorological aesthetics. Clouds are as ethereal as stars. Clouds put on a lavish daily show; this sets our moods: sunshine, a fine day, a rainy day, stormy weather.

Sunsets are paradigm meteorological aesthetics. Lingering into the twilight and evenings, we may experience both clouds and stars. Samuel Taylor Coleridge is gazing at the western evening sky:

And still I gaze—-and with how blank an eye!

And those thin clouds above, in flakes and bars, 
That give away their motion to the stars;

Those stars, that glide behind them or between, Now sparkling, now bedimmed, but always seen ...

I see, not feel, how beautiful they are. ${ }^{24}$

I can see the clouds above; they may interfere with my celestial view, obscuring the stars. Then again, the clouds too are part of the celestial view, over our heads. Consider how the moon in its phases is framed by high cirrus clouds and casts an ambience over the landscape.

Overhead is atmosphere. Air is invisible and so never visually aesthetic, though it may be heard and felt. Carbon, oxygen, nitrogen, hydrogen-four of the elements most essential for life are all in that atmosphere. There is an intricate relationship between the sky, the Earth, and those who inhabit the Earth. Thinking of the oxygen, hydrogen, carbon, nitrogen in the atmosphere returns us to our participatory connections with the heavens. The atmosphere, our kind of atmosphere, is as much a key to life as anything in the stars above. Yes, humans are fossil stardust, the output of the stars is necessary for life. But it is not sufficient for it. One needs these endless flows of water-laden air, of oxygen, nitrogen, carbon dioxide if there is to be life on Earth. Half of these stardust atoms now incorporated into my body once floated in these skies above me. The flow of water between Earth and sky determines the weather, the climate, and this has critically funded evolutionary natural history. Our sustainable future depends on it.

There is gaseous water in the atmosphere, invisible again, but the gaseous water condenses as visible droplets of water, clouds, in the aggregate most evidently visible. Clouds are a dominant feature of our everyday celestial aesthetics. ${ }^{25}$ Nowadays, in the air, we can often look down on the clouds, rather than up at them. Despite Shakespeare, clouds are never ugly. We do speak of a grey, overcast day. If the clouds are low enough we are in a fog and see only a few feet ahead. We may speak of threatening clouds, with a gathering storm on the horizon, but more often than not such clouds are aesthetically stimulating.

Meteorological aesthetics is, again, always and never the same. Yes, there are repeatedly the same types of clouds: cumulus, stratus, altocumulus, altostratus, cirrus - the wispy high ones. But within this typology, there is a mixture of order and disorder. Clouds may linger for hours, then again they can rapidly mutate from one form to another. This sky is reconstructed daily, even hourly. Clouds may figure prominently on our sense of landscape, as seen in landscape paintings. $^{26}$

Meteorology has turned out to be substantially more complex than anyone predicted. The circulations of the atmosphere are difficult to model. Witness the controversies surrounding global warming, and one of the more elusive features has been modeling the clouds. Chaos theory was born in meteorology and weather has proved impossible to predict more than two weeks ahead because of the chaotic elements in the atmospheric system. ${ }^{27}$

In views of Earth from space, we see also clouds, and on a scale of grandeur unavailable from the ground. Earth from space is not dark brown, as is earth, ground, but it is blue and white, and both are water, oceans and cloud. Even the continents are filtered though the blue mist. That mist, with its life support, is as 
vital to our being at home in the universe as are those stars in which the elements in our bodies are composed, or the sun which fuels our bodies.

\section{Above and below: At home in the universe}

Above us are those unthinkably vast distances, but then again it is we humans who do manage to think about such distances, measuring our universe in billions of light years. What is over our heads does get into our heads, not simply atoms from the atmosphere into our brains, but ideas about the sky and heavens above. The wonder is not just the vast astronomical distances; the wonder is as much this: Homo sapiens with mind enough to search the universe across the light years and back to its explosive origins. The wonder is that when we search so, we find that what is out there is inseparably linked with what is down here.

Those who turned eyes to the heaven were often looking for a sign. "Give us a sign!" They often took planetary conjunctions to be such an omen, although we now take such conjunctions to be an accident of cosmic perspective and to mean nothing at all. In fact, we may take the vast heavens to mean nothing at all, as did Steven Weinberg. But from still another perspective, maybe we do have a sign, or if not a sign, at least a connection.

There appeared in the twentieth century, another kind of celestial aesthetics: aesthetic experience not simply from below the celestial space above, but aesthetic experience in space. We got our heads in the clouds. Moreover, we got our heads into orbit, even got our heads past escape velocity. What astronauts see may be clearer astronomical views than those that can be had on the ground. They are often, somewhat surprisingly, views of Earth from space, which the astronauts by photography have shared with millions of us on the ground. Over 200 men and women astronauts from 20 nations unanimously report being earthstruck. Their photographs have been seen by over half the people on Earth, who, almost without exception, have found these whole Earth pictures aesthetically stimulating. Viewing Earthrise from the moon, the astronaut Edgar Mitchell, was entranced:

Suddenly from behind the rim of the moon, in long, slow-motion moments of immense majesty, there emerges a sparkling blue and white jewel, a light, delicate sky-blue sphere laced with slowly swirling veils of white, rising gradually like a small pearl in a thick sea of black mystery. It takes more than a moment to fully realize this is Earth ... home. ${ }^{28}$

A good planet is hard to find, and Earth is something of an anomaly, so far as we yet know. Most planets, even though they contain suitable elements, will not be in a habitable temperature zone. Located at a felicitous distance from the sun, Earth has liquid water, atmosphere, a suitable mix of elements, compounds, minerals, and an ample supply of energy. "It appears that Earth got it just right," conclude Peter D. Ward and Donald Brownlee. ${ }^{29}$

The heavens are celestial, majestic, sublime. But the heavens are also a world in which there is no caring about anything, life or death, the beautiful or the ugly. 
The heavens are the ultimate in indifference. Stars do not intend anything. Asteroids do not succeed or fail. There are no winners or losers. Nothing enjoys pleasure, suffers pain. Much less is there any aesthetic experience. There is no community on the lifeless moon, on Mars, Jupiter. Those places are comparatively, boring; indeed not until humans appear does either concept or percept of beautiful or boring arise.

Lost out there in the stars, and even though we alone have such aesthetic capacity, we may be moved by celestial vastness, immensity. But that too requires more perspective. The numbers in astronomy are huge, say, on the order of $10^{80}$, the number of protons in the visible universe. ${ }^{30}$ However, when amino acids are linked together to construct proteins, the possible structures are immense (often defined as greater than $10^{110}$ ) for proteins of approximately 100 amino acids; most proteins are over twice that long: "Because there are 20 different amino acids and a typical protein comprises some 200 of them, the number of possible proteins is $20^{200}$ [or about $10^{260}$ ].... All of the matter in the myriad galaxies of the universe falls far short of that required to construct but one example of each possible protein molecule." ${ }^{31}$ These huge numbers of logically possible permutations result from what is called combinatorial explosion.

The universe from big bang to present would have to be repeated $10^{67}$ times to create each one of these possible proteins just once. Typical DNA strands in mammals, with some hundred million base pairs, can be arranged in 10 raised to the $10^{\mathrm{s}}$ power different ways. ${ }^{32}$ There is an explosion of possibilities in complication. Many of these permutational possibilities could never be biologically functional, but a vast number could be. Nature on Earth rings the changes on these biomolecular possibilities, exploring biodiversity in adaptive fit. Consider in analogy the number of sentences that can be typed on a keyboard with 26 alphabetical letters, upper and lower case, some punctuation marks, spaces, numerals, a number in the range of 100 keys.

Astronomical nature and atomic nature, profound as they are, are nature-in-thesimple. At both ends of the spectrum of size, nature lacks the complexity that it demonstrates at the mesolevels, found in the earthen ecosystem, or at the psychological level in the human person. Astronomical nature is incredibly vast and energetic, but primitive. Such a statement will seem odd, on first reading, for the theories and calculations by which the mind probes such nature are among the most sophisticated known to science, as, for example, relativity theory and quantum mechanics. Physics is no simple science, and the stuff of its observations is abundantly mysterious. But that energetic matter, compared with life and mind, is as primitive as it is basic.

We encounter advanced forms of natural organization only at the middle ranges and in the other sciences. As a human person, I do not live at the range of the infinitely small, nor at that of the infinitely large, but we humans may well live at the range of the infinitely complex. If the DNA in the myriad cells of the human body were uncoiled and stretched out end to end, that microscopically slender thread would reach to the sun and back over half a dozen times.

I gaze at the night sky and think: the human being is the most sophisticated of known natural products. The human brain, built by DNA, is the most complex 


\section{Theology and Science}

entity known in the universe. In that brain, there takes place a mental combinatorial explosion superimposed not just on the physics and chemistry, but further on the biological combinatorial explosions in genetics. The human brain is capable of forming thoughts numbering something in the range of $10^{70,000,000,000}$ thoughts - a number that dwarfs the number of atoms in the visible universe $\left(10^{80}\right) .^{33}$ On a cosmic scale, humans are minuscule atoms, but on a complexity scale, humans have "hyperimmense" possibilities in mental complexity. ${ }^{34}$ In our 150 pounds of protoplasm, in our three pound brain is more operational organization than in the whole of the Andromeda galaxy.

I live out West, in panorama country. Montana is "big sky country." Colorado is where all the fourteeners are. I live where, on a clear day, you can see forever. Recently, I packed into the remote Bob Marshall Wilderness, and at night there is no light from civilization visible anywhere on the horizon. At night, you see ten times as many stars as you can from my backyard in town. A real part of that wilderness experience is the celestial aesthetics. Looking up, I found myself drawn to the pioneer experience:

How often at night, when the heavens were bright, With the light of the twinkling stars

Have I stood there amazed, and asked as I gazed, If their glory exceeds that of ours. ${ }^{35}$

I still wonder. The glory is both over our heads and in our heads.

\section{Endnotes}

1 E.A. Burtt, The Metaphysical Foundations of Modern Science (Atlantic Highlands, NJ: Humanities, 1996 [1952]), 238-239.

2 Alfred, Lord Tennyson, "Vastness," The Poems of Tennyson, ed. Christopher Ricks (New York: W.W. Norton, 1969 [1885]), 1346.

3 Karl Jaspers, The Origin and Goal of History (New Haven: Yale University, 1953), 237.

4 Steven Weinberg, The First Three Minutes (New York: Basic Books, 1988), 154.

5 Martin Rees, Our Cosmic Habitat (Princeton, NJ: Princeton University, 2001).

6 Wheeler interviewed in, Florence Helitzer, "The Princeton Galaxy," Intellectual Digest 3:10 (July 1973), 25-32, on p. 27.

7 Eugene P. Wigner, "The Unreasonable Effectiveness of Mathematics in the Natural Sciences," Communications on Pure and Applied Mathematics 13 (1960): 1-14, on pp. 2,14.

8 P.A.M. Dirac, "The Evolution of the Physicist's Picture of Nature," Scientific American 208:5 (1963): 45-53.

9 John Polkinghorne, Belief in God in an Age of Science (New Haven: Yale University, 1998), 2.

10 P.A.M. Dirac, "The Relation between Mathematics and Physics," Proceedings of the Royal Society (Edinburgh) 59 (1938-1939), Part II, 122-129, on p. 123.

11 Judith Wechsler, ed., On Aesthetics in Science (Cambridge, MA: MIT, 1978), 1, 6.

12 Irene Born. The Born-Einstein Letters, trans. Irene Born (New York: Walker and Company, 1971), 91.

13 Albert Einstein, Out of My Later Years, revised reprint edition (Westport, CT: Greenwood, 1970), 61.

14 Werner Heisenberg, Physics and Beyond (New York: Harper and Row, 1971), 68. 
15 Richard A. Kerr, "Europa vs. Titan," Science 322 (December 19, 2008): 1780-1781.

16 Francis Crick, What Mad Pursuit: A Personal View of Scientific Discovery (New York: Basic Books, 1988), 6,137-142.

17 Darwin quotes Herschel, "Letter to Charles Lyell, December 12, 1859," in Francis Darwin, The Life and Letters of Charles Darwin, 3 Vols. (London: John Murray, 1888), Vol. 2, 239-240.

18 James Watson, The Double Helix (New York: Atheneum, 1968), xi.

19 John D. Barrow, The Constants of Nature (New York: Pantheon Books, 2002), 113.

20 Paul Davies, Cosmic Jackpot: Why Our Universe is Just Right for Life (Boston: Houghton Mifflin, 2007).

21 Roger Penrose, The Road to Reality: A Complete Guide to the Laws of the Universe (New York: Alfred A. Knopf, 2005), 726.

22 Rees, Cosmic Habitat, 163.

23 William Shakespeare, King Richard II, Act 1, Scene 1.

24 Samuel Taylor Coleridge, "Dejection: An Ode" (1802), in English Romantic Poetry and Prose, ed. Russell Noyes (New York: Oxford University, 1956), 417-419.

25 Graeme L. Stephens, "The Useful Pursuit of Shadows," American Scientist 91 (2003): 442449.

26 J.E. Thornes, "Landscape and Clouds," The Geographical Magazine 51 (1979): 492-499; Hans Neuberger, "Climate in Art," Weather 25 (1970): 46-56; Kurt Badt, John Constable's Clouds (London: Routledge and Kegan Paul, 1950); Barbara Novak, "The Meteorlogical Vision: Clouds," in Nature and Culture: American Landscape and Painting 1825-1875 (New York: Oxford University, 1980), 78-100.

27 Edward N. Lorenz, "Climatic Determinism," Meteorological Monographs 8:30 (1968): 1-3.

28 Edgar Mitchell, quoted in Kevin W. Kelley, ed., The Home Planet (Reading, MA: Addison-Wesley, 1988), photograph 42.

29 Peter D. Ward and Donald Brownlee, Rare Earth: Why Complex Life Is Uncommon in the Universe (New York: Copernicus; Springer-Verlag, 2000), 265.

30 Barrow, Constants of Nature, 97-118.

31 Alwyn Scott, Neuroscience: A Mathematical Primer (New York: Springer-Verlag, 2002), 297.

32 Alwyn Scott, Stairway to the Mind: The Controversial New Science of Consciousness (New York: Copernicus; Springer-Verlag, 1995), 29-30.

33 Owen Flannagan, Consciousness Reconsidered (Cambridge, MA: MIT Press, 1992); Mike Holderness, "Think of a Number," New Scientist 170 (June 16, 2001): 45.

34 Scott, Stairway to the Mind, 81.

35 Brewster Higley, "Home on the Range" (1876).

\section{Biographical Notes}

Holmes Rolston, III is University Distinguished Professor at Colorado State University. He is the author of several books including Genes, Genesis and God (Cambridge University Press, 1999), Science and Religion: A Critical Survey (Templeton Press, 2006), and Environmental Ethics (Temple University Press, 1988). He has edited Biology, Ethics, and the Origins of Life (Wadsworth, 1997). 